\title{
Verhaltensgenetik
}

\section{Molekulare Grundlagen des} Vogelzugs

CORINNA LANGEBRAKE ${ }^{1,2}$, BRITTA S. MEYER ${ }^{1}$, MIRIAM LIEDVOGEL ${ }^{1,2}$

1 MAX PLANCK RESEARCH GROUP „VERHALTENSGENOMIK“, MAX-PLANCK-INSTITUT

FÜR EVOLUTIONSBIOLOGIE, PLÖN

2 INSTITUT FÜR VOGELFORSCHUNG „VOGELWARTE HELGOLAND”, WILHELMSHAVEN

\section{Migration is a complex behavior of which a significant proportion is} genetic. Which genes and epigenetic changes underpin migratory behavior remains largely unknown, but a revolution of sequencing technology, analytical approaches, and functional genomic tools promises rapid advances. We summarize our current understanding of the (epi)genetic architecture of seasonal traits and outline our vision of how technical developments and integrative approaches could be employed to identify and functionally validate candidate genes and regulatory elements in migratory species.

DOI: $10.1007 / \mathrm{s} 12268-021-1511-y$

(C) Die Autorinnen 2021

Der alljährlich zwischen Brutgebiet und Winterquartier stattfindende Vogelzug fasziniert durch seine Dimension, Präzision und Variabilität. Bei Singvögeln verlassen Altvögel die Brutquartiere häufig vor den Jungvögeln. Junge Singvögel finden also ohne die Hilfe der Eltern oder anderer Artgenossen über Kontinente hinweg mit beeindrucken-

der Präzision ihren Weg in ein Gebiet, in dem sie noch niemals zuvor waren. Wie schaffen sie das? Woher wissen sie wann, wohin und in welche Richtung sie fliegen müssen? Wie und woran orientieren sich Zugvögel? Trotz intensiver Forschung bleibt es spekulativ, wie und warum dieses Verhalten bei Vögeln entstanden ist und warum verschiedene

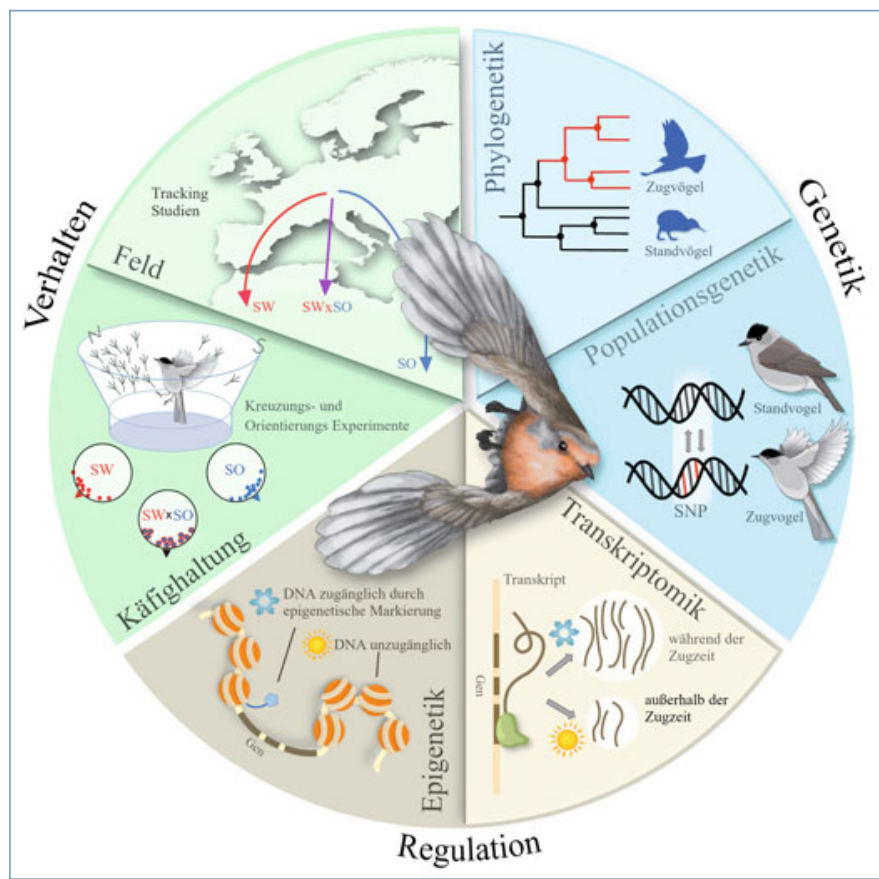
Arten und nicht selnogar b derselben Art ganz unterschiedliche Zugstrategien verfolgen. Die dispersal migration-Hypothese beschreibt den evo- lutionären Ursprung des Zugverhaltens derzeit am detailliertesten [1], da sie auf die variablen Eigenschaften dieses Verhaltens einzugehen vermag. Die Hypothese geht davon aus, dass zunächst neue Lebensräume durch eine Verbreitung ohne spezielle Richtungspräferenz durch Jungvögel kolonisiert wurden. Durch die schrittweise Verbreitung einer fiktiven Population sesshafter Vögel in saisonal geprägte Habitate oder klimatische Veränderungen erhöht sich der Selektionsdruck auf bestimmte Zugmerkmale. Mit stärker ausgeprägter Saisonalität und kalten Wintern im Brutgebiet stieg der Anteil ziehender Individuen innerhalb der Populationen im Laufe der Zeit entsprechend an. Laut dieser Hypothese liegt der Hauptgrund für die Evolution von Zugverhalten primär in der saisonal abhängigen Verteilung und Verarmung von Ressourcen sowie einer stark ausgeprägten Brutgebietstreue begründet [1, 2]. Phylogenetische Studien weisen auf unterschiedliche Ursprünge des Zugverhaltens hin, was dafür spricht, dass das Verhalten mehrere Male unabhängig voneinander entstanden ist. Wahrscheinlich wurden ubiquitär bei Vögeln vorhandene Gene im Laufe der Evolution für Anpassungen an den Vogelzug optimiert [3]. Beispielsweise besitzen alle Vögel eine genetische Uhr sowie die Fähigkeit zur Orientierung - und Zugvögel konnten beides jeweils für sich optimieren. Zugvögel verwenden die Sonne, Sterne und das Erdmagnetfeld als externe Kompassreferenzen zur Navigation und Orientierung und folgen einem genauen vererbten Zeitplan,

4 Abb. 1: Um die molekularen Grundlagen des Vogelzugs zu verstehen, ist eine integrative Zusammenarbeit verschiedener Disziplinen notwendig. Forschungsschwerpunkte bilden Genetik (blau), Regulation auf molekularer Ebene (braun) und das Verhalten der Tiere auf Ebene des Phänotyps (grün). Verhalten kann mithilfe von Daten-Loggern direkt in der Natur oder durch präzise Beobachtung unter kontrollierten Bedingungen in Käfigen charakterisiert werden (SO: Südost; SW: Südwest). Genetik: populationsgenetische und phylogenetische Methoden helfen, die Unterschiede innerhalb derselben Art bzw. die Evolutionsgeschichte von Zugmerkmalen und den zugrunde liegenden Genen zu entschlüsseln. Regulation: Unterschiede in Genexpressionsmuster können durch genetische Veränderung der DNA-Sequenz und/oder epigenetische Variation des chemischen Markierungsmusters der DNA moduliert werden. 
um artspezifische Überwinterungs- und Brutgebiete rechtzeitig zu erreichen [3].

Sobald die generelle Maschinerie einmal evolviert ist, kann eine Feinregulierung des Verhaltens durch verschiedene Umwelteinflüsse erfolgen [2]. Eiszeiten können beispielsweise als Auslöser für einen Wechsel vom Zug- zum Standvogel dienen, und ehemals ziehende Populationen durch eine Unterdrückung des Zugverhaltens zu Standvögeln werden. Dies darf nicht mit einem Verlust der genetischen Basis zu Zuganpassungen verwechselt werden, da das Verhalten bei veränderten klimatischen Bedingungen wieder aktiviert werden kann [3, 4]. Die Evolution des Zugverhaltens kann mittels phylogenetischer Methoden erforscht werden, sodass anhand der Merkmalsausprägung rezenter Arten auf die Vorfahren extrapoliert werden kann. Sobald Kandidatengene der Merkmalsausprägung bekannt sind, kann ein artübergreifender phylogenetischer Ansatz mit der genetischen Charakterisierung kombiniert und die Evolutionsgeschichte des Verhaltens mit der zugrunde liegenden genetischen Architektur verknüpft werden (Abb. 1, [5]).

Manche Arten befinden sich in einem evolutionären Übergangsstadium (Teilzieher), in dem alle Merkmalsausprägungen innerhalb einer Population beobachtet werden können. Teilzieherpopulationen zeigen eine hohe Variabilität des Zugverhaltens, z. B. etablierten sich in Populationen des amerikanischen Hausgimpels (Haemorhous mexicanus) innerhalb von 20 Jahren Zugvögel, nachdem hauptsächlich nicht ziehende Individuen aus Kalifornien in nördliche Habitate nach New York eingeführt wurden [6].

\section{Mönchsgrasmücke - ein ideales Modell}

Arten, die ein breites Spektrum des Zugverhaltens aufweisen, eignen sich hervorragend dafür, die Evolution des Verhaltens und die zugrunde liegenden molekularen Grundlagen zu erforschen (Abb. 2). Ein gutes Beispiel für hohe Variabilität im Zugverhaltens innerhalb ein- und derselben Art sind Mönchsgrasmücken (Sylvia atricapilla). Brutpopulationen in Nord- und Zentraleuropa sind Lang- und Mittelstreckenzieher, Populationen auf der Iberischen Halbinsel umfassen Kurzstreckenzieher und auch sesshafte Populationen. Das enorm variable Zugverhalten der Mönchsgrasmücke wurde in Selektions- und Kreuzungsexperimenten genutzt, um eine starke genetische Komponente des
Zugverhaltens aufzuzeigen. Mit gezieltem Selektionsdruck konnten Mönchsgrasmücken innerhalb weniger Generationen zu reinen Zug- oder Standvögeln gezüchtet werden [7, 8]. Zusätzlich zur Varianz in der Zugstrategie gibt es eine zentraleuropäische Zugscheide - ein Gebiet, in dem geografisch eng benachbarte Populationen unterschiedliche Zugrichtungen aufweisen: Populationen, die östlich der Zugscheide brüten, ziehen im Herbst südöstlich in ihre Überwinterungsgebiete, während Brutpopulationen westlich der Zugscheide südwestlich abziehen (Abb. 2, [9, 10]). Kreuzungsexperimente zeigen, dass Nachkommen von Eltern mit unterschiedlicher Zugorientierung eine intermediäre Route nach Süden wählen (Abb. 1, [11]). In Feldexperimenten konnte diese intermediäre Richtungspräferenz tatsächlich in der vermeintlichen Hybridzone entlang der Zugscheide bestätigt werden (Abb. 2, [10]). Seit den 1960er-Jahren beobachten wir darüber hinaus eine gänzlich neue Zugrichtung: Einige mitteleuropäische Mönchsgrasmücken ziehen im Herbst in nordwestliche Richtung und damit entgegen der ursprünglichen Richtungspräferenz in den wärmeren Süden - ein weiteres Beispiel, warum die Erforschung dieser Art so attraktiv ist [7, 9]. Diese neue Zugroute zu Überwinterungsgebieten in England hat sich vermutlich als Reaktion auf klimatische Veränderungen und bessere Ressourcenverteilung entwickelt (Abb. 2, schwarzer Pfeil, [9]). Die zugrunde liegenden Gene und Regulationssysteme des komplexen Zugverhaltens ausfindig zu machen, ist eine spannende Frage aktueller Forschungsprojekte.

\section{Methoden zur Charakterisierung des Verhaltens}

Unser Wissen über Unterschiede im Zugverhalten basiert bisher primär auf indirekter Charakterisierung, so wie klassischen Beringungsstudien oder Orientierungsexperimente im Labor. Zur Charakterisierung der individuellen Zugrichtung werden die Vögel in speziellen Orientierungstrichtern getestet (Käfighaltung, Abb. 1, [8, 11]). Mithilfe modernster Technik, den Helldunkelgeoloka- toren (kleine Speichereinheiten), die auf dem Rücken der Vögel befestigt werden und Lichtintensitätsdaten im Zeitverlauf aufzeichnen, ist es uns nun auch möglich, das Zugverhalten im Freiland zu charakterisieren. Dafür nutzen wir nach erfolgreichem Rückfang des Vogels die auf dem Lokator gespeicherten Daten, um anhand der longitudinalen und latitudinalen Positionen, die aus den zeitgekoppelten Lichtintensitätsdaten berechnet werden können, die Zugroute des Vogels zu rekonstruieren (Feldarbeit, Abb. 1, [10]).

\section{Molekulare Methoden}

Durch die enormen Fortschritte in der Hochdurchsatz-Sequenzierungstechnologie können wir mittlerweile Ergebnisse aus Verhaltensexperimenten direkt mit Analysen des gesamten Genoms verknüpfen. Mithilfe eines Referenzgenoms können so genomische Bereiche identifiziert werden, die bei der Modulierung des Verhaltens eine Rolle spielen. So können populationsgenomische Studien Hinweise auf Einzelnukleotid-Polymorphismen (SNPs) geben, die in regulatorischen Regionen von Kandidatengenen liegen und Hauptregulatoren des Zugverhaltens 


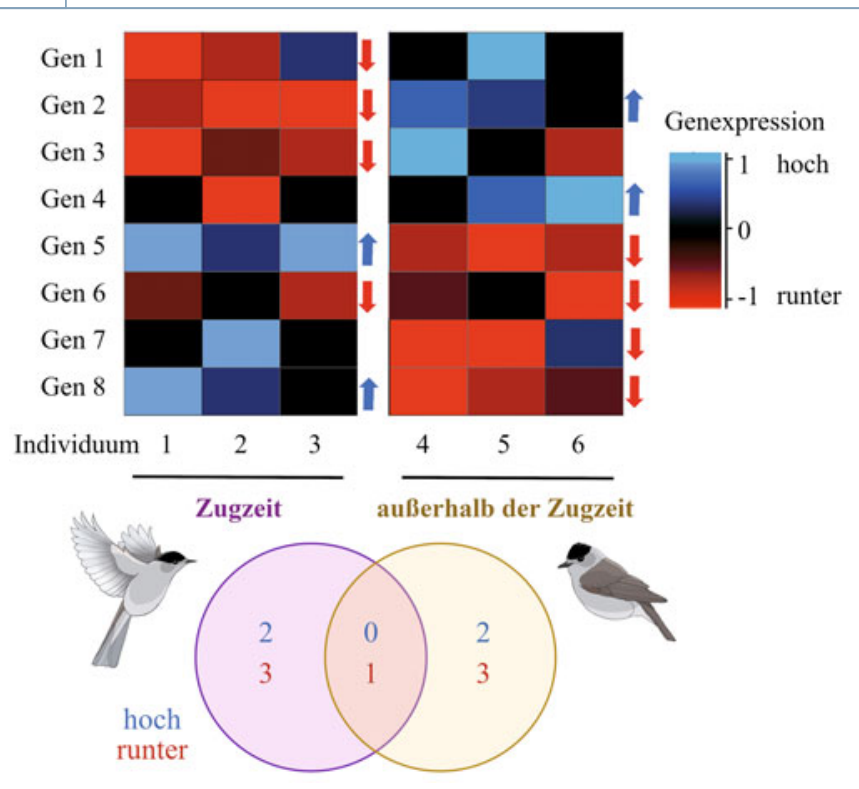

A Abb. 3: Durch RNAseq kann das Genexperessionsmuster von Zugvögeln während und außerhalb der Zugzeit charakterisiert werden. Ein Vergleich der zwei Gruppen kann Gene identifizieren, welche während der Zugzeit hoch- (blau) oder runterreguliert (rot) wurden. Die Ergebnisse können als Heatmap (oben) oder Venn-Diagramm (unten) dargestellt werden. In diesem fiktiven Beispiel wurden während der Zugzeit zwei Gene signifikant hochreguliert $($ Gen 5,8$)$ und drei Gene unterdrückt (Gen 1, 2, 3); eines dieser während der Zugzeit runterregulierten Gene (Gen 2) wurde außerhalb der Zugzeit verstärkt exprimiert. lierungsmuster werden beispielsweise mit Bisulfit-Sequenzierungsmethoden untersucht [12]. Hochregulierende oder unterdrückende Regulationsfaktoren können somit direkt mit dem variablen Aktivitätsmuster der durch sie kontrollierten Gene verknüpft werden, was ein sehr vielversprechender Ansatz ist, um Kandidatengene und regulatorische Bereiche de novo identifizieren zu können (Epigenetik, Abb. 1, Abb. 3).

\section{Fazit}

Durch die rasante Entwicklung neuer sein könnten [9]. Unterschiede in der DNASequenz müssen jedoch nicht die einzige Grundlage für variables Zugverhalten sein. Vergleiche des Genexpressionsmusters (z. B. RNAseq, Abb. 3) unterschiedlicher Gewebe während und außerhalb der Zugzeit liefern ebenfalls Hinweise auf Gene und regulatorische Gen-Netzwerke, die hierbei eine Rolle spielen können [12]. In unterschiedlichen Vogelarten wurden so während der Zugzeit unterschiedlich exprimierte Gene gefunden, die bei Stoffwechselaktivitäten (insbesondere Fettsäure- und Kohlenhydratstoffwechsel), Muskelwachstum und Neurogenese sowie der Kontrolle biologischer Rhythmen eine zentrale Rolle spielen (Abb. 3). Neuere Forschungsansätze in der Zugvogelforschung beziehen auch die Epigenetik mit ein, d. h. die Erforschung der Regulierung der Genexpression, bei der u. a. die DNA-Zugänglichkeit, die Histonmodifikation sowie die Bindung von Transkriptionsfaktoren an spezifische DNA-Sequenzen charakterisiert werden (cis-regulatorische Elemente bzw. Enhancer) [12]. Mögliche Methoden hierzu sind ChIP(chromatin immunoprecipitation)- oder ATAC(assay for transposase-accessible chromatin)-Seq [12]. Eine weitere wichtige epigenetische Modifikation, die typischerweise mit der Unterdrückung der Genexpression in Verbindung gebracht wird und damit Einfluss auf den Phänotyp haben kann, ist die DNA-Methylierung. DNA-Methy- und erschwinglicher Sequenzierungsmethoden haben sich neue Möglichkeiten in der evolutionären Verhaltensgenomik ergeben, die wir hier exemplarisch im Kontext der Zugvogelforschung aufzeigen. Erstmals wird es uns daher möglich sein, die verantwortlichen genomischen Regionen und molekularen Grundlagen für die Variation im Vogelzugverhalten identifizieren zu können, was bisher aufgrund der biologischen Komplexität in der Regulation von Verhaltensphänotypen weitgehend unbekannt blieb.

\section{Literatur}

[1] Salewski V, Bruderer B (2007) The evolution of bird migration - a synthesis. Sci Nat 94:268-279

[2] Winger BM, Auteri GG, Pegan TM, Weeks BC (2019) A long winter for the Red Queen: rethinking the evolution of seasonal migration. Biol Rev Camb Philos Soc 94:737-752 [3] Newton I (2007) The Migration Ecology of Birds. Academic Press, London

[4] Zink RM, Gardner AS (2017) Glaciation as a migratory switch. Sci Adv 3:e1603133

[5] Ramos JSL, Delmore KE, Liedvogel M (2017) Candidate genes for migration do not distinguish migratory and nonmigratory birds. J Comp Physiol A Neuroethol Sens Neural Behav Physiol 203:383-397

[6] Able KP, Belthoff JR (1998) Rapid "evolution” of migratory behaviour in the introduced house finch of eastern North

America. Proc Biol Sci 265:2063-2071

[7] Berthold P, Helbig AJ, Mohr G, Querner U (1992) Rapid Nature 360:668-670

[8] Helbig A (1996) Genetic basis, mode of inheritance and evolutionary changes of migratory directions in palaearctic warblers (Aves: Sylviidae). J Exp Biol 199:49-55 [9] Delmore K, Illera JC, Pérez-Tris J et al. (2020) The evolutionary history and genomics of European blackcap migration. eLife 9:247 microevolution of migratory behaviour in a wild bird species.
[10] Delmore KE, Van Doren BM, Conway GJ et al. (2020) Individual variability and versatility in an eco-evolutionary model of avian migration. Proc R Soc B 287:20201339 [11] Helbig AJ (1991) Inheritance of migratory direction in a bird species: a cross-breeding experiment with SE- and SW-migrating blackcaps (Sylvia atricapilla). Behav Ecol Sociobiol (1991) 28:9-12

[12] Buchberger E, Reis M, Lu T-H, Posnien N (2019) Cloudy with a Chance of Insights: Context Dependent Gene Regulation and Implications for Evolutionary Studies. Genes 10:492

Funding note: Open Access funding enabled and organized by Projekt DEAL. Open Access: Dieser Artikel wird unter der Creative Commons Namensnenn 4.0 International Lizenz veroffenticht, welche die Nutzung, Vervielfaltigun Bearbeitung, Verbreitung und Wiedergabe in jeglichem Medium und For erlaubt, sofern Sie den/die ursprünglichen Autor(en) und die Quelle
ordnungsgemäß nennen, einen Link zur Creative Commons Lizenz beifügen und ordnungsgemäß nennen, einen Link zur Creative Commons Lizenz beifu
angeben, ob Änderungen vorgenommen wurden. Die in diesem Artikel angeben, ob Anderungen vorgenommen wurden. Die in diesem Artikel
enthaltenen Bilder und sonstiges Drittmaterial unterliegen ebenfalls der genannten Creative Commons Lizenz, sofern sich aus der Abbildungslegende nichts anderes ergibt. Sofern das betreffende Material nicht unter der genannten Creative Commons Lizenz steht und die betreffende Handlung nich nach gesetzlichen Vorschriften erlaubt ist, ist für die oben aufgeführten Weiterverwendungen des Materials die Einwilligung des jeweiligen Rechteinhabers einzuholen. Weitere Details zur Lizenz entnehmen Sie bitte der

\section{Korrespondenzadresse:}

Prof. Dr. Miriam Liedvogel Institut für Vogelforschung An der Vogelwarte 21,

D-26386 Wilhelmshaven

miriam.liedvogel@ifv-vogelwarte.de
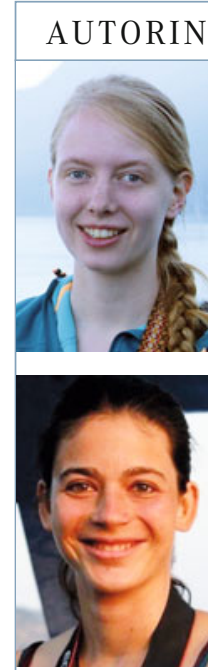

Britta Meyer

2014 Promotion an der Universität Basel, Schweiz. 2014-2018 Postdoc in der Marinen Evolutionsökologie, GEOMAR Helmholtz-Zentrum für Ozeanforschung, Kiel. Seit 2018 Postdoc in der MPRG Verhaltensgenomik, MPI für Evolutionsbiologie, Plön.

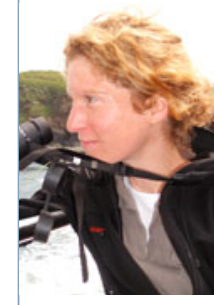

Miriam Liedvoge 2006 Promotion an der Universität Oldenburg. 20072009 Marie Curie Fellowship Oxford Universität, UK. 2010-2013 Feodor Lynen Fellowship Alexander-vonHumboldt-Stiftung, Lund Universität, Schweden und Feodor Lynen Reintegration Fellowship, Universität Freiburg. Seit 2015 Leiterin der MPRG Verhaltensgenomik am MPI für Evolutionsbiologie in Plön. 2020 Habilitation an der Universität Kiel. Seit 2020 Direktorin des IfV, Wilhelmshaven und Professorin für Ornithologie an der Universität Oldenburg. 